Annals of Pure and Applied Mathematics

Vol. 14, No. 2, 2017, 327-336

ISSN: 2279-087X (P), 2279-0888(online)

Published on 30 September 2017

www.researchmathsci.org

DOI: http://dx.doi.org/10.22457/apam.v14n2a16

Annals of

Pure and Applied

Mathematics

\title{
Solving Multi-Objective Optimization Problems through Unified Approach
}

\author{
H.A.Khalifa ${ }^{1}$ and N.M.Alharbi ${ }^{2}$

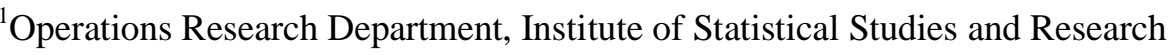 \\ Cairo University, Giza, Egypt \\ ${ }^{2}$ Mathematics Department, Sciences and Arts College in ARASS, Qassim University \\ Qassim, Saudi Arabia \\ Corresponding author. Email: hamiden_2008@yahoo.com
}

Received 19 August 2017; accepted 6 September 2017

\begin{abstract}
In this paper, unified approach for solving multi- objective optimization problem is introduced. The approach is based on the Reference Direction (RD) method introduced by Narula et al. [14], and the Attainable Reference Point (ARP) method introduced by Wang et al. [19]. This approach improves the performance of the ARP method by using the initial weak efficient solution of the RD method that is to improve the weights in the Lexicographic weighted Techebycheff program. The weights in the unified approach are constructed through the ARP and the weak efficient solution. A numerical example is given in the sake of the paper to clarify the obtained results.
\end{abstract}

Keywords: Multi- objective optimization problem; Efficient solution; Reference direction method ; Attainable reference point; Unified approach; Satisfactory solution

\section{AMS Mathematics Subject Classification (2010): 90C29}

\section{Introduction}

Multi-objective optimization problem (MOP) has become famous among many researchers due to more practical and realistic applications. In general, there is no single optimal solution in multi- objective problems, but rather a set of non- inferior (or pareto optimal) solutions from which the DM must select the most preferred solution as the one to implement (Cohon et al. [3]). The generation of the entire non- inferior solution set is not practical for most real world problems.

Most multi- objective methods are based on interaction between a decision maker (DM) and the mathematical model of the problem under consideration. A typical interactive method exhibits a hierarchical structure composed of an analysis level, which comprises the solution of some auxiliary single objective optimization problem, and decision level, at which the DM tries to induce the analysis level to generate a solution that optimizes his (her) preference function (Hwang and Masud [12], and Chankong and Haimes [2]).

Multi-objective optimization methods can be classified according to the DM influence in the optimization process (Hwang and Masud [12]):

1. Methods where DM does not provide information (no- preference methods) 


\section{H.A.Khalifa and N.M.Alharbi}

2. Methods where a posteriori information is used (posteriori methods)

3. Methods where a priori information is used (priori methods)

4. Methods where progressive information is used (interactive methods).

Since the pioneer interactive method for solving multi-objective nonlinear programming (MONLP) problem is given by Geoffrion et al. [9], many researchers have developed numerous interactive algorithms for solving multi- objective optimization problems (Aksoy et al. [1]) and Gradiner and Steuer [8]).

The main difference of the algorithms lies in their techniques for arriving to a final solution. Typically, a technique can be categorized into two classes (Sawaragi et al. [16]):

Class 1: An interactive optimization method, and

Class 2: An interactive satisfactory method

The Tchebycheff method is suggested in Steuer $[17,18]$ that the sampling of the pareto optimal set works in the most unbiased way if the ranges of the objective function values over the pareto optimal set are approximately the same. The reference point introduced by Wierzbicki [20] is based on a reference point aspiration levels. The reference point is a feasible or infeasible or desirable in the objective space which is reasonable desirable to the DM. The reference point is used to derive achievement scalarizing functions having minimal solutions at weakly, $\varepsilon$-properly or pareto optimal points. In this method, generating pareto optimal solutions is based on reference points, not on value functions or weighting vectors. No specific assumptions are based on the the problem to be solved. The satisficing trade- off method presented by Nakayama [13] is as follows:

After a weekly or a properly pareto solution has been obtained by optimizing a scalarizing function, it is presented to the DM. On the basis of this transformation(s) he (she) is used to classify the objective functions into three classes: the unacceptable objective function whose values he(she) wants to improve $\left(I^{<}\right)$, the acceptable objective whose values he(she) agrees to relax (impair) and the acceptable $\left(I^{>}\right)$objective functions whose values he(she) accepts as they are $\left(I^{*}\right)$ (such that $I^{=}=I^{<} \cup I^{>}$). The reference direction method presented by Narula et al. [14] is based on a reference directions, and it needs to minimize a series of achievement scalarizing problems at each iteration according to several values of the parameters $\alpha \quad(0 \leq \alpha \leq 1)$ according to the DM wishes. The solution of each achievement scalarizing problem is weakpareto optimal solution. To obtain only efficient solution, it is needed to solve a single objective surrogate problem. There is no stopping rule, and the iteration process stops according to the DM wishes. Wang et al. [19] proposed an interactive algorithm namely, the Attainable Reference Point method. This method uses the Lexicgraphic Weighted Techebycheff programming problem, in which the weighting vector is constructed through the Attainable Reference Point and the ideal point. This ensures the efficiency of the solution. The role of the DM is to compare the solution with the ideal point, and the reference point if the solution is not satisfactory he (she) chooses an unsatisfactory objective function and modify the reference values of the some satisfactory objectives. A new reference value for the unsatisfactory objective is reached after the solution of an auxiliary problem. Sadrabadi and Sadjadi [15] developed a new algorithm based on a new approach to solve the MOP by starting from utopian point, which is usually infeasible, and moving towards the feasible region via stepwise movement and a simple continuous 
Solving Multi-Objective Optimization Problems through Unified Approach

interaction with DM. De and Yadav [5] proposed an algorithm for solving multiobjective assignment problem through interactive fuzzy programming approach. Deshpande et al. [6] proposed a new method say, pareto front approximation method for multi-objective optimization problems with bound constraints. Fadil et al. [7] created a probabilistic multi- objective optimization model which described the aircraft trajectory uncertain. Das and Roy [4] proposed computational algorithm to solve MONLP problem with single valued neutrosophic data. Hakanen et al. [11] introduced efficient optimization techniques for solving multi- objective optimization problems arising for simulated moving bad processes. Ghaznaki et al. [10] studied multi- objective programming problem and proposed a scalarizing problem for it and also introduced the relation between the optimal solution of the scaralizing problem and the weakly efficient solution as properly efficient.

This paper deals with unified approach for solving multi- objective nonlinear programming problem. The approach is combined with the Reference Direction (RD) introduced by Narula et al. [14] and the Attainable Reference Point (ARP) method introduced by Wang et al. [19] In the unified approach, we still starting with a weak efficient solution as the first step and use the corresponding objective values to improve the weighting coefficients of the augmented Lexicographic Weighted Tchebycheff problem and hence modify the reference point in the case of an unsatisfactory solution for the decision maker (DM) he (she) wishes.

The remainder of the paper is organized as in the following five sections: In section2, multi- objective optimization problem (MOP) is introduced as specific definition and properties. In section 3, a unified approach for solving the MOP is presented. In section 4 , a numerical example is given to clarify the suggested unified approach introduced in section3. Finally, some concluding remarks are reported in section 5.

\section{Problem formulation and solution concept}

Consider the following multi- objective optimization problem

$$
\min _{x \in G} Z(x)=\left(f_{1}(x), f_{2}(x), \ldots, f_{k}(x)\right)^{T}
$$

Where, $x$ is a $n-$ dimensional vector of decision variables, $G \subset R^{n}$ is the feasible set of the decision variables, and $f_{i}(x), i=1,2,3, \ldots, k$ are $m$ distinct real -valued functions. Is assumed that:

1. The feasible region of decision variables $G \subset R^{n}$ is non- empty and compact.

2. $f_{i}(x), i=1,2,3, \ldots, k$, are continuous functions.

Definition 1. (Wang et al. [19]). Let $\bar{f}=\left(\bar{f}_{1}, \bar{f}_{2}, \ldots, \bar{f}_{k}\right)^{T}$ be an attainable reference point $\left(\bar{f}_{i} \geq f_{i}, i=1,2,3, \ldots, k\right.$ and $\bar{f}$ may be feasible or infeasible) for the problem (1), and $x^{*} \in G$.

1. $x^{*}$ is said to be reference efficient solution of problem (1) if $x^{*}$ is an efficient solution of problem (1) and $f\left(x^{*}\right) \leq \bar{f}$. 
H.A.Khalifa and N.M.Alharbi

2. A point $x^{*}$ is said to be satisfactory reference solution of problem (1) if $x^{*}$ is reference efficient solution of problem (1) and $f\left(x^{*}\right)$ is satisfactory for the DM.

\section{Unified approach}

The steps of the unified approach to obtain a satisfactory solution for the MOP (1) can be summarized as in the following steps:

Step 1: Calculate a starting point $x^{\wedge}$ that is by solving the following problem:

$\operatorname{Max} \lambda_{i}, i=1,2, \ldots, k$,

$$
\begin{aligned}
& \lambda_{i} \leq f_{i}(x) ; \forall i=1,2, \ldots, k, \\
& x \in G, 0 \leq \lambda_{i} \in R .
\end{aligned}
$$

Denote the corresponding objective vector by $f^{\wedge}$, go to step 2 .

Step 2: Give an initial reference point. DM specifies an initial reference point (Wang et al. (2001)), $\bar{f} \in R^{k}$ such that $\bar{f}_{i}^{o}>f_{i}^{\wedge}$ for every $i=1,2, \ldots, k$. Let $J=\{1,2, \ldots, k\}, J^{o}=J$. Let the iteration counter $h=0$, and go to step3.

Step 3: Search for a reference efficient solution. Let

$$
W_{i}^{h}=\frac{1}{\bar{f}_{i}^{h}-f_{i}^{\wedge}}, i=1,2,3, \ldots, k, \text { and }
$$

go to step 4 .

Step 4: Search for a reference non- efficient solution that is by solving the following Lexicographic Weighted Tchebycheff programming problem with the calculating weights and $f_{i}^{\wedge}, i=1,2, \ldots, k$, as

Lex $\min \left\{\alpha_{i},\left|f_{i}(x)-f_{i}^{\wedge}\right|\right\}, i=1,2,3, \ldots, k$

Subject to

$$
\begin{aligned}
& W_{i}^{h}\left|f_{i}(x)-f_{i}^{\wedge}\right| \leq \alpha_{i} ; \forall i=1,2,3, \ldots, k, \\
& x \in G, 0 \leq \alpha_{i} \in R
\end{aligned}
$$

To obtain an optimal solution $x^{h}$ and the corresponding objective vector $f^{h}$, and go to step 5 .

Step 5: The determination of termination. There exist three cases:

Case 1: If the DM is satisfied with the corresponding objective $f^{h}=f\left(x^{h}\right)$, stop- the satisfactory solution has been found by this method.

Case 2: If there is no satisfactory objective $f^{h}$, stop- there is no satisfactory solution can be found by this method. 
Solving Multi-Objective Optimization Problems through Unified Approach

Case 3: If there are some satisfactory objectives, ask the DM to select one of such objective and the amount $\Delta f$ to be sacrificed (decreased) in exchange for an improvement of some unsatisfactory objective.

Step 6: If $h=k$, stop- no satisfactory solution can be found by this method. Otherwise, set $h=h+1$, go to step 7 .

Step 7: The modification of the reference point

(i) The DM selects any $e_{h}$ in $J^{h}$ such that $f_{e_{h}}$ is an unsatisfactory objective in $\left\{f_{i}: i \in J^{h}\right\}$ at $f^{h}$. Let $J^{h+1}=J^{h} /\left\{e_{h}\right\}$. Separate $J^{h+1}$ into the following two parts:

Part 1: $J_{1}^{h}=\left\{i \in J^{h+1}: f_{i}^{h}<\bar{f}_{i}^{h}\right.$ and the DM wishes to release the value of $f_{i}$ at $\left.f^{h}\right\}$.

Part 2: $J_{2}^{h}=J^{h+1} / J_{1}^{h}$.

(ii) For $i \in J_{1}^{h}$ the DM presents $\Delta_{i}^{h}$, the amount to be relaxed for $f_{i}$ at $f^{h}$, such that $0<\Delta_{i}^{h} \leq \bar{f}_{i}^{h}-f_{i}^{h}$. Let $\bar{f}_{i}^{h+1}=f_{i}^{h}+\Delta_{i}^{h}$. For $i \in J^{h} / J^{h+1}$, let $\bar{f}_{i}^{h+1}=\bar{f}_{i}^{h}$.

(iii) In the case $\bar{f}_{i}^{h+1}=f_{i}^{h}$ for all $i \in J^{h} /\left\{e_{h}\right\}$, return to (i), to separate $J^{h+1}$ once again or return to (ii) to increase the amount to be relaxed for some $f_{i}\left(i \in J_{1}^{h}\right)$ at $f^{h}$, if the DM wishes to do so. In the case that $\bar{f}_{i}^{h+1} \neq f_{i}^{h}$ for some $i \in J^{h} /\left\{e_{h}\right\}$, go to (iv).

(iv) Let $e=e_{h}, f^{\prime}{ }_{i}=\bar{f}_{i}^{h+1}, i=1,2, \ldots, k, i \neq e_{h}$, and solve the following auxiliary problem

Min $f_{e}(x)$

Subject to

$$
\begin{aligned}
& f_{i}(x) \leq f^{\prime}{ }_{i}, i=1,2, \ldots, k, i \neq e \\
& x \in G .
\end{aligned}
$$

To obtain an optimal solution $x^{\prime h}$, when $f_{e_{h}}^{h^{h}}=f_{e_{h}}^{h}$ or $f_{e_{h}}^{\text {'h }}$ of objective $f_{e_{h}}$ is not satisfactory to the DM, return to (iii) to increase the amount to be relaxed for some $f_{i}\left(i \in J_{1}^{h}\right)$ at $f^{h}$ if the DM wishes. Otherwise, stop when $f_{e_{h}}^{t^{h}} \neq f_{e_{h}}^{h}$ and $f_{e_{h}}^{\text {th }}$ for objective $f_{e_{h}}$ is satisfactory to the DM he (she) provides $\Delta_{e_{h}}^{h}$, the larger the amount for $f_{e_{h}}$ to be improved at $f^{h}$, such that $0<\Delta_{e_{h}}^{h} \leq f_{e_{h}}^{h}-f_{e_{h}}^{h_{h}}$. Let $\bar{f}_{e_{h}}^{h+1}=f_{e_{h}}^{h}-\Delta_{e_{h}}^{h}$. 
H.A.Khalifa and N.M.Alharbi

(v) If $\bar{f}_{e_{h}}^{h+1}<f_{e_{h}}^{\prime h}, \quad$ let $h=h+1$ and return to (iii). Otherwise, let $x^{h+1}=x^{h} ; h=h+1$ and return to (iv) when $x^{\prime h}$ is unique optimal solution of the auxiliary problem (5) or let $x^{\text {th }}$ be an optimal solution of the following $\min _{i=1,2, \ldots, k} \alpha_{i}, \quad i=1,2, \ldots, k$

Subject to

$$
\begin{aligned}
& W_{i}^{h}\left|f_{i}(x)-f_{i}^{\wedge}\right| \leq \alpha_{i}, 1,2, \ldots, k, \\
& x \in G, 0<\alpha_{i} \in R .
\end{aligned}
$$

If $\bar{f}_{e_{h}}^{h+1} \geq f_{e_{h}}^{\text {hh }}$, let $h=h+1$, and return to step3.

Table 1. The advantages and disadvantages of ARP method, RD method, and Unified

\begin{tabular}{|c|c|c|}
\hline Method & Advantages & Disadvantages \\
\hline $\begin{array}{l}\text { Attainable } \\
\text { Reference } \\
\text { Point }\end{array}$ & $\begin{array}{l}\text { 1. It is a general method, that } \\
\text { is it is applicable to any } \\
\text { problem (differentiable or } \\
\text { non-differentiable, convex } \\
\text { or non convex). } \\
\text { 2. The iteration number is not } \\
\text { more than the objective } \\
\text { function numbers. } \\
\text { 3. The attainable reference } \\
\text { point may be feasible or } \\
\text { infeasible. } \\
\text { 4. The DM need not provide } \\
\text { the precise attainable } \\
\text { reference point in advance. } \\
\text { The DM modifies his } \\
\text { reference point under the } \\
\text { help of the method. } \\
\text { 5. The resulted solution is an } \\
\text { efficient solution. }\end{array}$ & $\begin{array}{l}\text { 1. It does not determine the } \\
\text { complete set of efficient } \\
\text { solutions. } \\
\text { 2. At each iteration step } \\
\text { through the modification of } \\
\text { the reference point, the DM } \\
\text { needs to solve an auxiliary } \\
\text { problem to guarantee its } \\
\text { attainability. } \\
\text { 3. The weights depend on the } \\
\text { ideal point and the } \\
\text { attainable reference point } \\
\text { through the use of the } \\
\text { Lexicographic weighted } \\
\text { Tchebycheff program. } \\
\text { 4. Through the modification of } \\
\text { the reference point, the DM } \\
\text { may need to return again } \\
\text { either to (ii) to increase the } \\
\text { amount to be relaxed for } \\
\text { some } f_{i} i \in J_{1}^{h}, \text { or to (i) to } \\
\text { separate } J^{h+1} \text { again . }\end{array}$ \\
\hline
\end{tabular}
approach 
Solving Multi-Objective Optimization Problems through Unified Approach

\begin{tabular}{|c|c|c|}
\hline $\begin{array}{l}\text { Reference } \\
\text { Direction }\end{array}$ & $\begin{array}{l}\text { 1. The algorithm starts with a } \\
\text { weak efficient solution. } \\
\text { 2. The solution of each } \\
\text { achievement scalarizing } \\
\text { problem is a feasible } \\
\text { solution for the successive } \\
\text { problem. }\end{array}$ & $\begin{array}{l}\text { 1. In each iteration, we need to } \\
\text { solve more than one } \\
\text { achievement scalarizing } \\
\text { problem according to the } \\
\text { wishes of the DM. } \\
\text { 2. The stopping rule is not } \\
\text { defined. It is according to the } \\
\text { DM wishes. } \\
\text { 3. The optimal solution of each } \\
\text { achievement scalarizing } \\
\text { problem is a weak efficient } \\
\text { solution of problem (1) and } \\
\text { to obtain only efficient } \\
\text { solution it is required to } \\
\text { solve a single objective } \\
\text { surrogate problem. } \\
\text { 4. It does not determine the } \\
\text { complete set of efficient } \\
\text { solutions. }\end{array}$ \\
\hline $\begin{array}{l}\text { Unified } \\
\text { Approach }\end{array}$ & $\begin{array}{l}\text { 1. It a general method, which } \\
\text { is applicable to any problem } \\
\text { (differentiable or non } \\
\text { differentiable, convex or } \\
\text { non convex) } \\
\text { 2. The iteration number is } \\
\text { surely less than the } \\
\text { objective numbers, hence } \\
\text { the computational effort } \\
\text { required to get the final } \\
\text { solution is considerably } \\
\text { reduced where the DM } \\
\text { reaches his/ her satisfactory } \\
\text { solution more rapidly than } \\
\text { the one obtained from the } \\
\text { attainable reference point. } \\
\text { 3. The ARP may be feasible or } \\
\text { infeasible. } \\
\text { 4. The DM need not provides } \\
\text { the precise attainable } \\
\text { reference point in advance, } \\
\text { but he needs only his } \\
\text { reference point under the } \\
\text { help of the method. } \\
\text { 5. The solution is efficient. }\end{array}$ & $\begin{array}{l}\text { 1. The method does not } \\
\text { introduce the complete set of } \\
\text { efficient solutions. } \\
\text { 2. Through the modification of } \\
\text { the reference point the DM } \\
\text { needs to solve an auxiliary } \\
\text { problem to guarantee } \\
\text { attainability and may need to } \\
\text { return again either to step (ii) } \\
\text { to increase the amount to be } \\
\text { relaxed for some } f_{i}, i \in J_{1}^{h} \\
\text { or to step (i) to separate } J^{h+1} \\
\text { again. }\end{array}$ \\
\hline
\end{tabular}




\section{H.A.Khalifa and N.M.Alharbi}

\section{Numerical example}

Consider the following problem

$$
\begin{aligned}
& \min Z(x)=\left(f_{1}(x), f_{2}(x)\right)^{T} \\
& \text { Subject to } \\
& \qquad \begin{array}{l}
\left(x_{1}-3\right)^{2}+\left(x_{2}-2\right)^{2} \leq 0, \\
2 x_{1}-x_{2} \geq 0 .
\end{array}
\end{aligned}
$$

where, $f_{1}(x)=x_{1}$ and $f_{2}(x)=x_{2}$.

Let us apply the steps of the unified procedure as:

Step 1: Starting with $x^{\wedge}$ by solving the following problem:

$$
\begin{aligned}
& \underset{i=1,2}{\operatorname{Max}} \lambda_{i} \\
& \text { Subject to } \\
& \lambda_{1} \leq x_{1}, \\
& \lambda_{2} \leq x_{2}, \\
& \left(x_{1}-3\right)^{2}+\left(x_{2}-2\right)^{2} \leq 0 \text {, } \\
& 2 x_{1}-x_{2} \geq 0 \text {, } \\
& 0<\lambda_{i} \in R, i=1,2 \text {. }
\end{aligned}
$$

Let the solution be $x^{\wedge}=(3,0019,1.9967)$ and the corresponding objective values are $f^{\wedge}=(3,0019,1,9967)$.

Step 2: DM specifies an initial attainable reference point $\bar{f} \in R^{k}$, such that $\bar{f}_{i}>f_{i}^{\wedge}$ for every $i=1,2$, that is

$3.0004<\bar{f}_{1}<5.0004 ? 5.0004$, and $1.9967<\bar{f}_{2}<4.0004 ? 4.0004$.

Then, $\bar{f}=(5.0004,4.0004)^{T}$.

Let $J=\{1,2\},, J^{O}=J$. Set the iteration counter $h=0$, and go to step3.

Step 3: Calculate the weighting vector from the following relation:

$$
W_{1}^{1}=\frac{1}{\bar{f}_{1}-f_{1}^{\wedge}}=0.5004, \text { and } W_{2}^{1}=\frac{1}{\bar{f}_{2}-f_{2}^{\wedge}}=0.4991
$$

Step 4: Solve the Lexicographic Weighted Tchebycheff problem with the calculating weights and $f^{\wedge}$ according to the problem (4) as

$$
\min \left\{\alpha_{i},\left|x_{1}-3.0019\right|+\left|x_{2}-1.9967\right|\right\}
$$


Solving Multi-Objective Optimization Problems through Unified Approach

Subject to

$$
\begin{aligned}
& 0.5004\left|x_{1}-3.0019\right| \leq \alpha_{1}, \\
& 0.4991\left|x_{2}-1.9967\right| \leq \alpha_{2}, \\
& \left(x_{1}-3\right)^{2}+\left(x_{2}-2\right)^{2} \leq 0 \\
& 2 x_{1}-x_{2} \geq 0 \\
& x_{1}, x_{2} \geq 0,0 \leq \alpha_{i} \in R, i=1,2 .
\end{aligned}
$$

Step 5: Case1: $x^{*}=(3.0019,0), f^{*}=(3.0019,0), \bar{f}=(5.0004,4,0004)$, and $f^{\wedge}=(3.0019,1.9967)$ is the DM satisfied Y/N: Y, stop with $x^{*}=(3.0019,0)$ and $f^{*}=(3.0091,0)$ as the final solution.

\section{Concluding remarks}

In this paper, we have suggested unified approach for solving multi- objective optimization problems. This approach based on the combined with the Reference Direction method introduced by Narula et al. [14], and the Attainable Reference Point (ARP) method introduced by Wang et al. [19], and adapted the Lexicographic Weighted Tcheycheff program. The main development we made constitutes in using the weak efficient solution obtained from the initial step of the reference direction method to improve the weights in the Lexicographic Weighted Tcheycheff program. This improvement reduced the number of iterations, and hence the computational effort required to obtain the final solution has been reduced.

Acknowledgment. We grateful to the anonymous referee for his valuable suggestion to organize, and modify the paper.

\section{REFERENCES}

1. Y.Aksoy, T.W.Butler and E.D.Minor, Comparative studies in interactive multiobjective mathematical programming, European Journal of Operations Research, 89 (1996) 408-422.

2. V.Chankong and Y.Y.Haimes, Multi-objective Decision- Making: Theory and Methodology, North Holland and Series in System Science and Engineering, 1983.

3. L.J.Cohon, J.R.Current and C.S.Revelle, An interactive approach to identify the best compromise solution for two objectives shortest path problems, Computer \& Operations Research, 17(2) (1990) 187-198.

4. P.Das and T.K.Roy, Multi-objective non-linear programming problem based on neutrosophic optimization technique and its application in riser design problem, Neutrosophic Sets and Systems, 9 (2015) 88- 95.

5. P.K.De and B.Yadav, An algorithm to solve multi-objective assignment problem using interactive fuzzy goal programming approach, Int. J. Contemp. Math. Science, 6 (2011) 1651-1662.

6. S.Deshpande, T.Watson, T.Layne and A.R.Canfield, Multi-objective optimization using an adaptive weighting scheme, Optimization Methods and Software, 31(1) (2016) 110-133. 
H.A.Khalifa and N.M.Alharbi

7. P.Fadil, B.Abou El Majd, H.Rahil, E.Ghazi and N.Kaabouch, Multi-objective optimization approach for air traffic flow management, MATEC Web of Conference, 105 0005, 2017.

8. R.L.Gardiner and R.E.Steuer, Unified interactive multiple objective programming, European Journal of Operational Research, 74 (1994) 391-406.

9. M.A.Geoffrion, J.S.Dyer and A. Feinberg, An interactive approach for multi-criteria optimization with an application to the operation of an academic department, Management Science, 18(4) (1972) 357-368.

10. M.Ghaznaki, M.Itati and E.Khomam, An interactive algorithm for solving multiobjecttive optimization problems based on a general scaralization technique, Iranian Journal of Numerical Analysis and Optimization, 6(1) (2016)79- 99.

11. J.Hakanen, Y.Kawajiri, K.Miettinen, and L.T.Biegler, Interactive multi-objective optimization for simulated moving bed processes, Control and Cybernetics, 36(2) (2007)283- 302.

12. L.C.Hwang and A.S.Masud, Multiple Objective Decision Making: Method and Applications, Springer, New York, 1979.

13. H.Nakayama, Aspiration level approach to interactive multi-objective programming and its applications, Advances in Multicriteria Analysis, Edited by P.M.Pardalos, Y.Siskos, C.Zopounidis, Kluwer Academic Publishers, Dordrecht, 1995.

14. C.S.Narula, L.Kirilov and V.Vassiley, An interactive algorithm for solving multiple objective nonlinear programming problems, Multiple Criteria Decision Making, and Proceeding of the tenth International Conference: Expand and Enrich the Domain of Thinking and Application, Springer Verlag, Berlin, 1993.

15. M.R.Sadrabadi and S.J.Sadjadi, A new interactive method to solve multiobjective linear programming problems, Scientific Research, 4 (2009) 237-247.

16. Y.S.Sawaragi, H.Nakayaaman and T.Tanino, Theory of Multi-objective, Academic Press, New York, 1985.

17. E.R.Steuer, Multiple Criteria Optimization: Theory, Computation, and Applications, John Wiley \& Sons, Inc., 1986.

18. E.R.Steuer, The Tchebycheff procedure of interactive multiple objective program mming, Multiple Criteria Decision Making and Risk Analysis Using Microcomputers, Edited by B. Karapak, S.Zionts, Springer- Verlag, Berlin, Heidelberg, 1989.

19. M.X.Wang, Z.L.Qin and Y.D.Hu, An interactive algorithm for solving multi-criteria decision making: the attainable reference point method, IEEE Transaction on Systems, Man, and Cybernetics-Part A: Systems and Humans, 31(3) (2001)194-198.

20. L.Wierzbicki, The use of reference objective in multi-objective optimization, in G.Fandel and T.Gal (Eds), Multiple Criteria Decision Making Theory and Application, Springer-Verlag, New York, 1980. 